Volume: 2, Issue: 4, December 2017, Pages: 229-234, DOI: http://dx.doi.org/10.19082/ah229

\title{
EVALUATION OF PREDICTIVE FACTORS OF DEVELOPMENT OF TYPE 2 DIABETES MELLITUS (T2DM) IN PATIENTS WITH GESTATIONAL DIABETES MELLITUS (GDM)
}

\author{
Masoumeh Razmpoor ${ }^{1}$, Masoumeh Kheirandish ${ }^{2}$, Shahram Zare $^{3}$
}

1: M.D., Infectious and Tropical Diseases Research Center, Hormozgan Health Institute, Hormozgan University of Medical Sciences, Bandar Abbas, Iran

2: M.D., Assistant Professor, Endocrinology and Metabolism Research Center, Hormozgan University of Medical Science, Bandar Abbas, Iran

3: Ph.D., Professor of Department of statistic, Hormozgan University of Medical Sciences, Bandar Abbas, Iran

\section{TYPE OF ARTICLE: ORIGINAL ARTICLE}

\begin{abstract}
Introduction: Several groups have been considered as the population at risk for diabetes, one of which is women with a history of gestational diabetes. Women with gestational diabetes are likely to develop type 2 diabetes mellitus (T2DM) and cardiovascular disease. Given the great importance of controlling risk factors for T2DM following gestational diabetes, it was decided to conduct a study to investigate predictive factors of the development of T2DM in patients with gestational diabetes mellitus (GDM).

Methods: This cross-sectional study had been conducted on pregnant women with gestational diabetes in 2015. All pregnant women underwent screening for gestational diabetes at the first visit. At six to 12 weeks after parturition, they were put under oral glucose tolerance test (OGTT) again. Follow-up was done in all cases where there was an abnormality in diagnostic test or symptoms of hyperglycemia, glycosuria, and abnormal weight gain. All blood samples were centrifuged, and plasma samples obtained were transferred to measure glucose. Chi-square test was used for data analysis with IBM SPSS 21.0 statistical software. $P$-value less than 0.05 was considered as the significance level.

Results: A total of approximately 185 patients were included in this study with a mean age of $27.7 \pm 4.8$ years. Among 185 patients who were followed-up for six to 12 weeks after parturition, $10 \%$ of patients were with type 2 diabetes after pregnancy. In addition, 10\% of postpartum patients have had impaired glucose tolerance test (IGT), but diabetes was not found. Also, 30.27\% $(\mathrm{n}=56)$ of the patients have had impaired fasting glucose (IFG). Finally, about $44.32 \%(n=82)$ of the patients had abnormal blood glucose test (AGT). After analyzing the data, it was found that, of 185 patients, two patients had a BMI less than 20, three people with a BMI between 20-24.9, seven patients with a BMI between 25-29.9, and three patients with BMI between 30-40 had diabetes, according to GTT test. Risk factors such as cholesterol, history of hyperthyroidism, history of hypothyroidism, polycystic ovarian syndrome, obesity, acanthosis nigricans, and family history of diabetes had no significant relationship with development of diabetes after pregnancy $(\mathrm{p}>0.05)$.

Conclusion: About $10 \%$ of patients after parturition clearly had diabetes, according to the tests carried out. Our study showed that BMI and FBS play a significant role in predicting the development of diabetes, but factors such as history of miscarriage, stillbirth, macrosomia, death without cause and medical records such as hypertension, obesity, hyperthyroidism, and hypothyroidism have not played a significant role in this prediction.

KEYWORDS: Gestational diabetes mellitus, Diabetes, Type 2 diabetes mellitus, Impaired fasting glucose
\end{abstract}

\section{INTRODUCTION}

The incidence of diabetes suddenly or the first time during pregnancy is called gestational diabetes (1). Pregnancy by intermediaries such as growth hormones, corticotropin-secreting hormones, placental lactogen, and progesterone induces insulin resistance (2). Insulin resistance during pregnancy if the pancreas does not have sufficient capacity to neutralize it has progressed and becomes disease. The prevalence due to differences in screening tests and diagnostic criteria and personal characteristics such as age and body mass index (BMI) are different. Prevalence is

\section{Correspondence:}

Dr. Masoumeh Razmpoor. Infectious and Tropical Diseases Research Center, Hormozgan Health Institute, Hormozgan University of Medical Sciences, Bandar Abbas, Iran.

Tel: +989173617674, Email: masoomeh_r57@yahoo.com

Received: February 29, 2017, Accepted: June 30, 2017, Published: December 2017

iThenticate screening: February 30, 2017, English editing: July 8, 2017, Quality control: July 12, 2017

(C) 2017 The Authors. This is an open access article under the terms of the Creative Commons Attribution-NonCommercialNoDerivs License, which permits use and distribution in any medium, provided the original work is properly cited, the use is non-commercial and no modifications or adaptations are made. 
constantly rising, which is probably a reason for the increase of the average maternal age and weight (3-7). Overall prevalence of gestational diabetes mellitus (GDM) in Iran in the recent years has been reported from $1.3 \%$ to $8.9 \%$, which is the rate in the city of Bandar Abbas on the basis of Carpenter's Coustan criteria.

Several groups have been considered as the population at risk of diabetes, one of which includes women with a history of gestational diabetes (8). It is known that many of these women are afflicted with overt diabetes in later years $(8,9)$. Women with gestational diabetes are likely to develop type 2 diabetes and cardiovascular disease increases; thus, their infants are more at risk of developing long-term complications (10-12). Early detection of diabetes can prevent long-term complications such as nephropathy and retinopathy. Fetal complications such as chromosomal abnormalities, etc. can also be prevented $(13,14)$. Sivaraman et al. have reported the risk of developing type 2 diabetes in women who had a history of gestational diabetes after 5 years at $6.9 \%$ and after 10 years at $21.1 \%$ (15). In another study, weight gain, hyperlipidemia and hypertension, and a family history of diabetes were not significantly correlated with gestational diabetes development, but high BMI and FBS has highly been correlated with diabetes development (16).

M. Ekelund et al. reported that women who have had abnormal blood glucose levels during pregnancy have assigned higher levels of fasting blood sugar after pregnancy. In this study, a significant relationship between increasing levels of BMI and diabetes after pregnancy was observed (17). Treatment of diabetes and its acute and chronic complications requires significant spending by patients and the healthcare system; further, disability due to this disease is a source of considerable damage at the community level. Therefore, early detection of the disease plays an important role in reducing complications and disability caused by them $(18,19)$. The aim of this study was evaluate the predictive factors of development of type 2 diabetes mellitus (T2DM) in patients with GDM.

\section{MATERIALS AND METHODS}

\subsection{Research Design and Setting}

This cross-sectional study was carried out between January and December 2015 in Hormozgan province, Iran.This study started with evaluating patients with gestational diabetes referred to hospitals of Shahid Mohammadi and Shariati and also to the researcher's office; then, about 15 patients were excluded due to the unavailability or lack of cooperation. These patients were evaluated again in approximately six to 12 weeks after birth.

\subsection{Participants}

Inclusion criteria: All pregnant women with gestational diabetes who consent to participate in the study.

Exclusion criteria: Patients who did not consent to participate in the study; patients who had diabetes before pregnancy.

\subsection{Data Collection}

In the first visit for prenatal care, all pregnant women were evaluated for gestational diabetes with screening methods. In the case of normality, this test was repeated again in weeks 28-24 of pregnancy. After six to 12 weeks after delivery oral glucose tolerance test (OGTT) was checked. In all cases, where there was an impairment in diagnostic tests or symptoms of hyperglycemia, glycosuria, and abnormal weight gain, the follow-up was done. Screening test was used in a $50 \mathrm{~g}$ and 1-hour glucose challenge test (GCT) with the criterion of glucose equal to or more than $135 \mathrm{mg} / \mathrm{dl}$. In the case of impairment in this test, follow-up or three-hour glucose tolerance OGTT 75 test was conducted for diagnosis. To do a 2-hour glucose tolerance test, it has been advised for three days of preparation, including the use of at least $150 \mathrm{~g}$ of carbohydrates per day, and the test was carried out on the fourth day after 8 hours of fasting. To perform the test, three blood samples were taken from a person at $0,1,2$ hours after ingestion of $75 \mathrm{~g}$ glucose. All blood samples were centrifuged, and plasma samples obtained were transferred to the laboratory to measure glucose. The blood glucose measurement method, glucose oxidase, and the auto-analyzer were used. GDM diagnostic standard test was at least one impairment in the diagnosis test. Diagnosis of diabetes and impaired glucose tolerance was done in accordance with the America Diabetes Association. Fasting blood sugar over $126 \mathrm{mg} / \mathrm{dl}$, a random sample of blood sugar above $200 \mathrm{mg} / \mathrm{dl}$ or 2-hour blood glucose higher than $200 \mathrm{mg}$ / $\mathrm{dl}$ after OGTT75 and HbA1C confirmed the diagnosis of diabetes. Fasting glucose levels between 110-126 or 2hour blood sugar after eating was considered between 140-199 mg / dl 199-140 as impaired glucose tolerance. 


\subsection{Research Ethics}

This study was conducted under the supervision of Hormozgan University of Medical Sciences. All patient information is entered into the confidential questionnaire with no name, and all questionnaires were coded. The study is approved by ethics committee of Hormozgan University of Medical Sciences.

\subsection{Statistical Analysis}

This study is a descriptive study; all data were entered into IBM SPSS 21.0 statistical software and were analyzed using descriptive statistics (mean and frequency) and statistical tests. Also, chi-square test was used to compare the frequency of each of the factors examined in the study groups. $P$-value less than 0.05 was considered as the significance level.

\section{RESULTS}

\subsection{Demographic Data}

Among the total of approximately 185 patients enrolled in this study, all of whom were women, their mean age was $27.7 \pm 4.8$ years. Of the 185 patients who entered the study $(11.8 \%), 22$ patients had BMI less than $20(54.3 \%)$, and 101 patients had BMI between 20-24.9 (29.6\%), 55 patients had BMI between 25-29.9 (4.3\%), and eight patients had BMI higher than 30.

\subsection{Main Results}

The study found that, among 185 patients who were followed-up for six to 12 weeks after the parturition 16 patients $(8.6 \%)$ had type 2 diabetes after pregnancy, and diabetes development in these patients was shown. On the other hand, $10 \%$ (19 patients) of patients had impaired glucose tolerance test (IGT) after parturition, but diabetes was not seen. Furthermore, 56 patients (30.27\%) showed impaired fasting glucose (IFG). Finally, about 82 patients ( $44.32 \%$ ) had abnormal blood glucose test (AGT). It was found that, of 185 patients, two patients who had a BMI less than 20, three people with a BMI between 20-24.9, seven people with a BMI between 25-29.9, and three patients with a postpartum BMI between 30-40 were included in diabetes GTT test. Eighty two patients who had fasting blood sugar less than 92 in pregnancy were not diabetic after pregnancy by GTT criteria. Also, of approximately 102 patients with impaired fasting glucose, 15 (15\%) patients were with diabetic GTT criteria, and only one patient was with fasting plasma glucose higher than the 126 who had been diagnosed with diabetes after delivery $(\mathrm{p}<0 / 001)$. It was also observed that, among 82 patients who had FBS less than 92 during pregnancy, about 72 patients $(87.8 \%$ ) had normal fasting blood sugar levels after pregnancy, and 102 patients, who had FBS between 92-125 during pregnancy, had impaired postpartum fasting glucose; also, one patient with blood sugar more than 126 during pregnancy demonstrated impaired fasting glucose in post-pregnancy GTT test $(\mathrm{p}<0 / 001)$. Risk factors such as cholesterol, history of hyperthyroidism, history of hypothyroidism, polycystic ovarian syndrome, obesity, acanthosis nigricans, and family history of diabetes had no significant relationship with development of diabetes after pregnancy $(\mathrm{p}>0.05)$ (Table 1$)$.

Table 1: Development of type 2 diabetes mellitus (T2DM) in patients with gestational diabetes mellitus (GDM) based on risk factors

\begin{tabular}{|c|c|c|c|c|c|}
\hline \multicolumn{2}{|l|}{ Risk factor } & Positive & Negative & Total & $P$-value \\
\hline \multirow[t]{2}{*}{ History of abortion } & Positive & $6(8.5)$ & $65(91.5)$ & 71 & \multirow{2}{*}{0.893} \\
\hline & Negative & $9(7.9)$ & $105(92.5)$ & 114 & \\
\hline \multirow[t]{2}{*}{ History of Macrosomia } & Positive & $1(3.8)$ & $25(96.2)$ & 26 & \multirow[t]{2}{*}{0.390} \\
\hline & Negative & $14(8.8)$ & $145(91.2)$ & 159 & \\
\hline \multirow[t]{2}{*}{ History of stillbirth } & Positive & $1(4)$ & $24(96)$ & 25 & \multirow[t]{2}{*}{0.418} \\
\hline & Negative & $14(8.8)$ & $146(91.3)$ & 160 & \\
\hline \multirow[t]{2}{*}{ History of neonatal death } & Positive & $1(3.6)$ & $27(96.4)$ & 28 & \multirow[t]{2}{*}{0.340} \\
\hline & Negative & $14(8.9)$ & $143(91.9)$ & 157 & \\
\hline \multirow[t]{2}{*}{ History of weight gain } & Positive & $3(7.9)$ & $35(92.1)$ & 38 & \multirow[t]{2}{*}{0.957} \\
\hline & Negative & $12(8.2)$ & $135(91.8)$ & 147 & \\
\hline \multirow[t]{2}{*}{ History of diabetes } & Positive & $0(0)$ & $0(0)$ & 0 & \multirow[t]{2}{*}{0.893} \\
\hline & Negative & $15(8.1)$ & $170(91.9)$ & 185 & \\
\hline \multirow[t]{2}{*}{ Hypertension } & Positive & $0(0)$ & $10(100)$ & 10 & \multirow[t]{2}{*}{0.390} \\
\hline & Negative & $15(8.6)$ & $160(91.4)$ & 175 & \\
\hline Cholesterol background & Positive & $1(4.8)$ & $20(95.2)$ & 21 & 0.551 \\
\hline
\end{tabular}




\begin{tabular}{|c|c|c|c|c|c|}
\hline & Negative & $14(8.5)$ & $150(91.5)$ & 164 & \\
\hline \multirow[t]{2}{*}{ History of hypothyroidism } & Positive & $5(13.2)$ & $33(86.8)$ & 38 & \multirow[t]{2}{*}{0.201} \\
\hline & Negative & $10(6.8)$ & $137(93.2)$ & 147 & \\
\hline \multirow[t]{2}{*}{ History of hyperthyroidism } & Positive & $0(0)$ & $5(100)$ & 5 & \multirow[t]{2}{*}{0.501} \\
\hline & Negative & $15(8.3)$ & $170(91.9)$ & 180 & \\
\hline \multirow[t]{2}{*}{ History of polycystic ovary } & Positive & $0(0)$ & $29(100)$ & 29 & \multirow[t]{2}{*}{0.082} \\
\hline & Negative & $15(9.6)$ & $141(90.4)$ & 156 & \\
\hline \multirow[t]{2}{*}{ Obesity background } & Positive & $1(3.6)$ & $27(96.4)$ & 28 & \multirow[t]{2}{*}{0.340} \\
\hline & Negative & $14(8.9)$ & $143(91.9)$ & 157 & \\
\hline \multirow[t]{2}{*}{ Nigricans background } & Positive & $1(16.7)$ & $5(83.3)$ & 6 & \multirow[t]{2}{*}{0.435} \\
\hline & Negative & $14(7.8)$ & $165(92.5)$ & 179 & \\
\hline \multirow[t]{2}{*}{ Family history of diabetes } & Positive & $13(10.2)$ & $115(89.8)$ & 128 & \multirow[t]{2}{*}{0.126} \\
\hline & Negative & $2(3.5)$ & $55(96.5)$ & 57 & \\
\hline
\end{tabular}

\section{DISCUSSION}

In the current study, predictive factors of type 2 diabetes development in patients with gestational diabetes has been discussed. A total of approximately 185 patients of the 200 patients who entered the study remained after loss due to lack of availability or inappropriate cooperation. Of these, about $10 \%$ of patients were with gestational diabetes after pregnancy clearly during tests carried out. Our study showed that BMI and FBS play a full role in predicting diabetes development, but factors such as history of miscarriage, stillbirth, macrosomia, birth and death without due, and medical records indicating hypertension, obesity, hyperthyroidism and hypothyroidism have not played a significant role in this prediction.

In the study, conducted BMI and diabetes development has shown a significant relationship; also, women who had higher fasting blood glucose levels during pregnancy have experienced impaired fasting glucose after pregnancy. According to Schaefer-Graf et al., no significant relationship between diabetes and BMI was found; thus, perhaps the higher prevalence of obesity can be noted as the reason in the study, while impaired fasting glucose was consistent with diabetes development in our study. On the other hand, in our study, history of neonatal death did not show a significant relationship. The reasons for this difference can be due to the smaller sample size of our study compared with this study and having a control group in this study (20). Schaefer-Graf et al. conducted another study that examines the factors involved in postpartum diabetes and how to reduce its patients (21). This study has shown that BMI is closely associated with the incidence of postpartum diabetes. This is also expressed in our study. The study has also shown that high levels of blood sugar in pregnant women can risk developing diabetes or impaired glucose tolerance after pregnancy because, in women who have experienced a higher level of FBS during pregnancy, there has been impaired fasting glucose after pregnancy or diabetes; thus, this relationship proved significant (21).

A history of gestational diabetes, macrosomia, frequency of pregnancy, family history of diabetes, and maternal age did not show a significant relationship with postpartum diabetes; in our study, they have shown no significant relationship. It is worth noting that the development of diabetes in patients in this study was about two times our study (20\%); of course, this difference has also obvious reasons. Perhaps the reasons for this difference are the different design of the study and longer follow-up after the pregnancy. The reasons for similarity in some results in the sample size can be noted (21).

Another study conducted by Merce Albareda et al. on 696 women with gestational diabetes history showed that the risk of diabetes was about $13.8 \%$ and the risk of glucose intolerance was about $42.4 \%$. These numbers in our study were about $10 \%$ and $35.6 \%$. Also, BMI was significantly correlated with diabetes in this study; by increasing it, the risk of diabetes or impaired glucose tolerance has been increased. In our study, BMI also plays a significant role in predicting glucose intolerance and diabetes. Macrosomia history, frequency of pregnancy and gestational age, as in our study, also had a significant relationship in this study. But what about the family history of diabetes, this relationship has been significant in that this variable is not significant in our study. Among the causes of this difference, the high volume of samples in this study can be noted. Also, the existence of control group in took much longer follow-up of patients (about 7 years) and racial differences between the subjects in this study and our study may explain different outcomes (22). 
Ekelund et al. also showed $30 \%$ of women with diabetes and glucose intolerance at about $50 \%$. The results of this study showed that high levels of FBS and BMI play an effective role in the development of diabetes, which was consistent with our results (17). What is clear from the analysis of other studies and our study is that high levels of blood sugar during pregnancy is certainly effective and useful in predicting and forecasting the development of diabetes after gestational diabetes. In almost all studies, this factor has been the main factor, although, along with the level FBS, BMI is one of the top helpful items in this prediction, but most patients have been able to predict FBS and have allocated more meaningful relationships. Other variables such as abortion, stillbirth, neonatal macrosomia, abnormal weight gain, hypertension, hyperlipidemia, hypothyroidism, and hyperthyroidism and have not had a significant relationship with diabetes after pregnancy; perhaps, as the causes of these outcomes, are the low sample size of the study and the loss of some samples and short follow-up period of patients included. There are disadvantages of this study, e.g., the more homogeneous sample size can have a beneficial effect in the analysis of the effects of these variables.

\section{CONCLUSIONS}

According to the results of this study and previous studies, the most important factor in predicting the development of type 2 diabetes in pregnancy is a high level of fasting blood sugar. Other factors evaluated in this study do not have a significant relationship, but more studies are needed to prove ineffectiveness or the effectiveness of these variables. It is suggested that, due to the significant prevalence of gestational diabetes and the possible development of postpartum diabetes, further studies with larger sample sizes and longer follow-up are necessary.

\section{ACKNOWLEDGMENTS:}

The paper is extracted from the results of a thesis by Dr. Masoumeh Razmpoor for the degree of specialist in internal medicine. The authors want to thank to directors of Hormozgan University of Medical Sciences for their help and support.

\section{CONFLICT OF INTEREST:}

The authors declare that they have no conflict of interests.

\section{AUTHORS' CONTRIBUTIONS:}

All authors contributed to this project and article equally. All authors read and approved the final manuscript.

\section{REFERENCES:}

1) International Association of D, Pregnancy Study Groups Consensus P, Metzger BE, Gabbe SG, Persson B, Buchanan TA, et al. International association of diabetes and pregnancy study groups recommendations on the diagnosis and classification of hyperglycemia in pregnancy. Diabetes Care. 2010 Mar; 33(3): 676-82. DOI: 10.2337/dc09-1848. PMID: 20190296. PMCID: PMC2827530.

2) Proceedings of the 4th International Workshop-Conference on Gestational Diabetes Mellitus. Chicago, Illinois, USA. 14-16 March 1997. Diabetes Care. 1998 Aug; 21 Suppl 2: B1-167. PMID: 9841138.

3) Moyer VA, Force USPST. Screening for gestational diabetes mellitus: U.S. Preventive Services Task Force recommendation statement. Ann Intern Med. 2014 Mar 18; 160(6): 414-20. PMID: 24424622.

4) Ferrara A. Increasing prevalence of gestational diabetes mellitus: a public health perspective. Diabetes Care. 2007 Jul; 30 Suppl 2: S141-6. PMID: 17596462.

5) Dabelea D, Snell-Bergeon JK, Hartsfield CL, Bischoff KJ, Hamman RF, McDuffie RS, et al. Increasing prevalence of gestational diabetes mellitus (GDM) over time and by birth cohort: Kaiser Permanente of Colorado GDM Screening Program. Diabetes Care. 2005 Mar; 28(3): 579-84. PMID: 15735191.

6) Kim SY, Saraiva C, Curtis M, Wilson HG, Troyan J, Sharma AJ. Fraction of gestational diabetes mellitus attributable to overweight and obesity by race/ethnicity, California, 2007-2009. Am J Public Health. 2013 Oct; 103(10): e65-72. PMID: 23947320. PMCID: PMC3780749.

7) Grant WB. Comment on: Bardenheier et al. Variation in prevalence of gestational diabetes mellitus among hospital discharges for obstetric delivery across 23 states in the United States. Diabetes Care 2013; 36: 1209-1214. Diabetes Care. 2013 Jul; 36(7): e102. PMID: 23801804. PMCID: PMC3687291.

8) American Diabetes A. Diagnosis and classification of diabetes mellitus. Diabetes Care. 2011 Jan; 34 Suppl 1: S62-9. PMID: 21193628 . PMCID: PMC3006051.

9) Krishnaveni GV, Hill JC, Veena SR, Geetha S, Jayakumar MN, Karat CL, et al. Gestational diabetes and the incidence of diabetes in the 5 years following the index pregnancy in South Indian women. Diabetes Res Clin Pract. 2007 Dec; 78(3): 398-404. PMID: 17640759. PMCID: PMC2358951. 
10) Jensen DM, Korsholm L, Ovesen P, Beck-Nielsen H, Molsted-Pedersen L, Damm P. Adverse pregnancy outcome in women with mild glucose intolerance: is there a clinically meaningful threshold value for glucose? Acta Obstet Gynecol Scand. 2008; 87(1): 59-62. PMID: 18158628.

11) Sermer M, Naylor CD, Gare DJ, Kenshole AB, Ritchie JW, Farine D, et al. Impact of increasing carbohydrate intolerance on maternal-fetal outcomes in 3637 women without gestational diabetes. The Toronto Tri-Hospital Gestational Diabetes Project. Am J Obstet Gynecol. 1995 Jul; 173(1): 146-56. DOI: 0.1016/0002-9378(95)90183-3. PMID: 7631672.

12) Sacks DA, Greenspoon JS, Abu-Fadil S, Henry HM, Wolde-Tsadik G, Yao JF. Toward universal criteria for gestational diabetes: the 75-gram glucose tolerance test in pregnancy. Am J Obstet Gynecol. 1995 Feb; 172(2 Pt 1): 607-14. PMID: 7856693.

13) Expert Committee on the D, Classification of Diabetes M. Report of the expert committee on the diagnosis and classification of diabetes mellitus. Diabetes Care. 2003 Jan; 26 Suppl 1: S5-20. PMID: 12502614.

14) Lamar ME, Kuehl TJ, Cooney AT, Gayle LJ, Holleman S, Allen SR. Jelly beans as an alternative to a fiftygram glucose beverage for gestational diabetes screening. Am J Obstet Gynecol. 1999 Nov; 181(5 Pt 1): 1154-7. PMID: 10561636.

15) Sivaraman SC, Vinnamala S, Jenkins D. Gestational diabetes and future risk of diabetes. J Clin Med Res. 2013 Apr; 5(2): 92-6. PMID: 23519363. PMCID: PMC3601506.

16) Lin $\mathrm{CH}$, Wen SF, Wu YH, Huang YY, Huang MJ. The postpartum metabolic outcome of women with previous gestational diabetes mellitus. Chang Gung Med J. 2005 Nov; 28(11): 794-800. PMID: 16422186.

17) Ekelund M, Shaat N, Almgren P, Groop L, Berntorp K. Prediction of postpartum diabetes in women with gestational diabetes mellitus. Diabetologia. 2010 Mar; 53(3): 452-7. PMID: 19957074.

18) Glatthaar C, Welborn TA, Stenhouse NS, Garcia-Webb P. Diabetes and impaired glucose tolerance. A prevalence estimate based on the Busselton 1981 survey. Med J Aust. 1985 Nov 11; 143(10): 436-40. PMID: 4088108.

19) The World Health Report 1997--conquering suffering, enriching humanity. World Health Forum. 1997; 18(3-4): 248-60. PMID: 9478137.

20) Schaefer-Graf UM, Buchanan TA, Xiang AH, Peters RK, Kjos SL. Clinical predictors for a high risk for the development of diabetes mellitus in the early puerperium in women with recent gestational diabetes mellitus. Am J Obstet Gynecol. 2002 Apr; 186(4): 751-6. DOI: 10.1067/mob.2002.121895. PMID: 11967502.

21) Schaefer-Graf UM, Klavehn S, Hartmann R, Kleinwechter H, Demandt N, Sorger M, et al. How do we reduce the number of cases of missed postpartum diabetes in women with recent gestational diabetes mellitus? Diabetes Care. 2009 Nov; 32(11): 1960-4. DOI: 10.2337/diacare.26.4.1199. PMID: 19641163. PMCID: PMC2768188.

22) Albareda M, Caballero A, Badell G, Piquer S, Ortiz A, de Leiva A, et al. Diabetes and abnormal glucose tolerance in women with previous gestational diabetes. Diabetes Care. 2003 Apr; 26(4): 1199-205. PMID: 12663597. 